\title{
Subspecific Differentiation of Mycobacterium avium Complex Strains by Automated Sequencing of a Region of the Gene (hsp65) Encoding a 65-Kilodalton Heat Shock Protein
}

\author{
DOUGLAS S. SWANSON, ${ }^{1,2}$ VIVEK KAPUR, ${ }^{2} \dagger$ KATHRYN STOCKBAUER, ${ }^{2}$ XI PAN, ${ }^{2}$ \\ RICHARD FROTHINGHAM, ${ }^{3,4}$ AND JAMES M. MUSSER ${ }^{2,5 *}$ \\ Section of Infectious Diseases, Department of Pediatrics, ${ }^{1}$ and Section of Molecular Pathobiology, Department of \\ Pathology, ${ }^{2}$ Baylor College of Medicine, and Clinical Microbiology Laboratory and Molecular Diagnostics Laboratory, \\ The Methodist Hospital, ${ }^{5}$ Houston, Texas 77030; Infectious Disease Section, Durham Veterans Affairs Medical Center, \\ Durham, North Carolina 27705; ; and Division of Infectious Diseases and International Health, Department of \\ Medicine, Duke University Medical Center, Durham, North Carolina $27710^{4}$
}

\begin{abstract}
To develop a strategy for rapid species assignment and strain differentiation of Mycobacterium avium complex (MAC) organisms, the sequence of a 360-bp region of the gene (hsp65) encoding a 65-kDa heat shock protein was determined for 56 isolates, including 21 patient isolates and 35 reference strains. Eleven $h s p 65$ alleles were identified, and there was no sharing of alleles between strains classified as $M$. avium and Mycobacterium intracellulare based on serovar and species-specific DNA hybridization probes. Phylogenetic analysis showed that 30 strains had one of two $h s p 65$ alleles which were found in known $M$. avium organisms, 23 strains had one of six alleles allied with known $M$. intracellulare organisms, and three MAC isolates had one of three $h s p 65$ alleles that differed substantially from the consensus $M$. avium and $M$. intracellulare hsp65 sequences. Estimates of strain relationships based on the sequences of $h s p 55$ and the 16S-23S ribosomal DNA internal transcribed spacer were similar. Automated DNA sequencing of a 360-bp region of the $h s p 65$ gene from MAC organisms provides a rapid and unambiguous marker system for strain differentiation and permits specific assignment of these acid-fast organisms for diagnostic purposes.
\end{abstract}

The bacteria of the Mycobacterium avium complex (MAC) are a group of related acid-fast organisms that are abundant in nature $(21,51)$. This complex is comprised of two principal species (Mycobacterium avium and Mycobacterium intracellulare) containing morphologically and biochemically indistinguishable organisms (21). Members of the MAC are opportunistic pathogens of animals and humans that can be isolated from environmental sources, such as water, soil, plants, and house dust $(12,20,33,46,52)$. These bacteria have long been known to cause pulmonary infections in adults and cervical lymphadenitis in children. More recently, they have gained considerable importance as a frequent cause of disseminated disease and death in AIDS patients $(15,22,51)$. For example, 25 to $50 \%$ of adult AIDS patients and 10 to $15 \%$ of pediatric AIDS patients in the United States are infected with MAC (19, $21,27,30$ ). These individuals have much lower survival rates than AIDS patients without MAC infections $(4,17,18)$.

The epidemiology of MAC infections is poorly defined, in part due to a lack of unambiguous and rapid strain-specific molecular markers. The standard technique used for MAC strain differentiation has been serologic typing of surface molecules, and 28 serovars have been described. Nucleic acid and antibody probes and high-performance liquid chromatography (HPLC) analysis have been used to assign serovars 1 to 6,8 to 11 , and 21 to $M$. avium and serovars 7,12 to 20 , and 25 to $M$. intracellulare (36). Serovar 27 and perhaps serovar 26 are considered to be Mycobacterium scrofulaceum serovars (50). The

\footnotetext{
* Corresponding author. Mailing address: Department of Pathology, Baylor College of Medicine, One Baylor Plaza, Houston, TX 77030. Phone: (713) 798-4198. Fax: (713) 798-4595. E-mail: jmusser@path .bcm.tmc.edu.

$\dagger$ Present address: Department of Veterinary Pathobiology, University of Minnesota, St. Paul, MN 55108.
}

specific status of the remaining serovars is undetermined (36, $50)$.

Several other strategies have been utilized for differentiating MAC strains. These strategies include phage typing $(5,7)$, analysis of restriction fragment length polymorphism (RFLP) profiles of plasmids $(6,28)$, chromosomal DNA $(1,3,9,28)$, a 360 -bp fragment of the $h s p 65$ gene (43), and insertion elements $(12,13,25,26,31,35,45)$, multilocus enzyme electrophoresis analysis $(47,54)$, nucleotide sequencing of the gene encoding $16 \mathrm{~S}$ rRNA and the internal transcribed spacer (ITS) located between the genes encoding $16 \mathrm{~S}$ and $23 \mathrm{~S}$ rRNAs $(2,8,10,11$, $24,34,40,48)$, and analysis with commercial probes, such as the Accuprobes specific for $M$. avium and $M$. intracellulare (Gen-Probe, San Diego, Calif.).

Recently, using automated DNA sequence analysis of a 360-bp segment of the gene ( $h s p 65$ ) encoding a $65-\mathrm{kDa}$ heat shock protein, workers identified eight allelic variants among 11 MAC organisms (23). This finding suggested the possibility that $h s p 65$ polymorphism could be exploited for molecular diagnosis of MAC infections. In order to assess the utility of species assignment and subspecific differentiation of MAC strains by characterization of hsp 65 allele polymorphism, we studied sequence variation in 56 MAC strains, including reference organisms and bacteria recovered from human infections. Nucleotide variations in the target segment provided an unambiguous, high-resolution system for strain differentiation and species assignment.

\section{MATERIALS AND METHODS}

Bacterial isolates. The 56 isolates studied were obtained from a collection held by one of us (R.F.); these isolates have been previously characterized to identify sequence polymorphisms in the 16S-23S ribosomal DNA (rDNA) ITS $(10,11)$. A total of 35 of the isolates are laboratory reference organisms, and 21 of the isolates were cultured from clinical specimens. The Trudeau Mycobacterial Culture Collection and American Type Culture Collection accession numbers for the following 35 reference strains have been published previously (10): TMC 706, 
TABLE 1. Characteristics of 33 MAC strains studied

\begin{tabular}{|c|c|c|c|c|c|c|c|}
\hline \multirow[b]{2}{*}{ Strain $^{a}$} & \multirow[b]{2}{*}{$\begin{array}{l}\text { Consensus } \\
\text { serovar }\end{array}$} & \multicolumn{3}{|c|}{ Species as determined by ${ }^{b}$ : } & \multirow[b]{2}{*}{ Consensus species } & \multirow[b]{2}{*}{$\begin{array}{c}\text { ITS } \\
\text { allele }\end{array}$} & \multirow[b]{2}{*}{$\begin{array}{l}\text { hsp } 65 \\
\text { allele }\end{array}$} \\
\hline & & $\begin{array}{c}\text { T-catalase } \\
\text { serotype method }\end{array}$ & $\begin{array}{l}\text { HPLC pattern } \\
\text { method }\end{array}$ & $\begin{array}{c}\text { Gen-Probe } \\
\text { hybridization } \\
\text { method }\end{array}$ & & & \\
\hline B-92 & 1 & M. avium & M. avium & M. avium & M. avium & Mav-A & hsp65.1 \\
\hline $14141-1395$ & 2 & M. avium & M. avium & M. avium & M. avium & Mav-A & hsp65.1 \\
\hline 6195 & 3 & M. avium & $M$. intracellulare & M. avium & M. avium & Mav-A & hsp65.1 \\
\hline 34540-Wales & 6 & M. avium & M. avium & M. avium & M. avium & Mav-A & hsp65.1 \\
\hline $13528-1079$ & 4 & M. avium & M. avium & M. avium & M. avium & Mav-B & hsp65.1 \\
\hline SJB \#2 & 8 & M. avium & $M$. avium & M. avium & M. avium & Mav-B & hsp65.1 \\
\hline 2993 & 21 & M. avium & M. avium & M. avium & M. avium & Mav-B & hsp65.1 \\
\hline 6194 & 2 & M. avium & $M$. intracellulare & M. avium & M. avium & Mav-C & hsp65.1 \\
\hline $17584-286$ & 9 & M. avium & M. avium & M. avium & M. avium & Mav-D & hsp65.1 \\
\hline $11907-300$ & $\mathrm{NC}^{c}$ & M. avium & M. avium & M. àvium & M. avium & Mav-A & hsp65.2 \\
\hline TMC 1463 & 4 & M. avium & M. avium & M. avium & M. avium & Mav-A & hsp65.2 \\
\hline $14186-1424$ & 11 & M. avium & M. avium & M. avium & M. avium & Mav-A & hsp65.2 \\
\hline $25546-759$ & 5 & M. avium & M. avium & M. avium & M. avium & Mav-B & hsp65.2 \\
\hline TMC 1461 & 10 & M. avium & M. avium & M. avium & M. avium & Mav-B & hsp65.2 \\
\hline $1602-1965$ & 10 & M. avium & M. avium & M. avium & M. avium & Mav-B & hsp65.2 \\
\hline P-42 & 12 & M. intracellulare & M. intracellulare & $M$. intracellulare & $M$. intracellulare & Min-A & hsp65.3 \\
\hline ATCC 25122 & 13 & M. intracellulare & M. intracellulare & M. intracellulare & M. intracellulare & Min-A & hsp65.3 \\
\hline Edgar Boone & 14 & M. intracellulare & M. intracellulare & M. intracellulare & M. intracellulare & Min-A & hsp65.3 \\
\hline TMC 1406 & 16 & M. intracellulare & M. intracellulare & M. intracellulare & $M$. intracellulare & Min-A & hsp65.3 \\
\hline P-54 & 17 & M. intracellulare & $M$. intracellulare & $M$. intracellulare & $M$. intracellulare & Min-A & hsp65.3 \\
\hline TMC 1419 & 20 & M. intracellulare & M. intracellulare & M. intracellulare & $M$. intracellulare & Min-A & hsp65.3 \\
\hline $72-888$ & 25 & M. intracellulare & M. intracellulare & $M$. intracellulare & M. intracellulare & Min-A & hsp65.3 \\
\hline 1244 Hillberry & 26 & M. intracellulare & $M$. intracellulare & $M$. intracellulare & M. intracellulare & Min-A & hsp65.3 \\
\hline 6845 & 12 & $M$. intracellulare & $M$. intracellulare & M. intracellulare & M. intracellulare & Min-A & hsp65.3 \\
\hline 157-Manten & 7 & M. intracellulare & M. intracellulare & M. intracellulare & $M$. intracellulare & Min-A & hsp65.4 \\
\hline Yandle & 16 & M. intracellulare & M. intracellulare & M. intracellulare & M. intracellulare & Min-A & hsp65.4 \\
\hline TMC 1473 & 15 & $M$. intracellulare & $M$. intracellulare & M. intracellulare & M. intracellulare & Min-A & hsp65.5 \\
\hline 12645 & 23 & M. intracellulare & M. intracellulare & $\mathrm{NR}^{d}$ & M. intracellulare & MAC-B & hsp65.6 \\
\hline 23393 & 23 & M. intracellulare & M. intracellulare & M. avium & M. intracellulare & MAC-C & hsp 65.6 \\
\hline Melnick & 18 & $\mathrm{ND}^{e}$ & M. intracellulare & NR & $\mathrm{NC}$ & MAC-D & hsp65.7 \\
\hline W-552 & 19 & $M$. intracellulare & M. intracellulare & M. intracellulare & $M$. intracellulare & MAC-A & $h s p 65.9$ \\
\hline P-49 & 7 & M. scrofulaceum & M. scrofulaceum & NR & M. scrofulaceum & MAC-E & $h s p 65.11$ \\
\hline 5154-O'Connor & 22 & $M$. intracellulare & Unknown & NR & $\mathrm{NC}$ & MAC-F & hsp65.13 \\
\hline
\end{tabular}

${ }^{a}$ Strains are described in references 10 and 11; the descriptions include accession numbers for strains belonging to the Trudeau Mycobacterial Culture Collection and the American Type Culture Collection.

${ }^{b}$ Serovar designations and species assignments based on T-catalase probe and Gen-Probe hybridization analysis results and HPLC patterns of mycolic acids and consensus species are described in reference 50 .

${ }^{c} \mathrm{NC}$, no consensus.

${ }^{d} \mathrm{NR}$, no reaction with any of the probes tested.

${ }^{e} \mathrm{ND}$, not done.

the $M$. avium type strain, B-92, 14141-1395, 6195, 34540-Wales, 13528-1079, SJB \#2, 2993, 6194, TMC 1463, 11907-300, 17584-286, 14186-1424, 25546-759, TMC 1461, 1602-1965, P-42, ATCC 25122, Edgar Boone, TMC 1406, P-54, TMC 1419 , 72-888, 1244 Hillberry, 6845, 157-Manten, Yandle, TMC 1473, 12645, 23393, Melnick, W-552, P-49, and 5154-O'Connor. The clinical isolates were identified as members of the MAC by performing a DNA probe analysis with Accuprobes (Gen-Probe) and were cultured from patients 1 through 18 and 20 as described previously (11). In Table 1, the results of seroagglutination tests, Gen-Probe hybridization, a T-catalase antibody probe analysis, and an analysis of the HPLC patterns of mycolic acids as reported in a multicenter study by the International Working Group on Mycobacterial Taxonomy (IWGMT) (50) for 33 of the 35 reference strains are compared with the allele assignment results obtained from ITS and $h s p 65$ sequence analyses.

Automated sequencing of $h s p 65$ DNA. DNA was obtained from the organisms by lysing the bacteria with glass beads in a Minibeadbeater (Biospec Products, Bartlesville, Okla.) as described elsewhere $(10,11)$. A 441-bp segment (nucleo- tides 396 to 836 ) (38) of hsp65 was amplified by PCR as described previously (23). Briefly, the target sequence was amplified with a DNA thermal cycle (Perkin-Elmer Cetus, Norwalk, Conn.) by using the following oligonucleotide primers: forward primer Tb11, (5'-ACCAACGATGGTGTGTCCAT-3') and reverse primer Tb12 (5'-CTTGTCGAACCGCATACCCT-3'). The thermal cycler parameters used were 30 cycles consisting of denaturation at $94^{\circ} \mathrm{C}$ for $1 \mathrm{~min}$, annealing at $60^{\circ} \mathrm{C}$ for $1 \mathrm{~min}$, and extension at $72^{\circ} \mathrm{C}$ for $1 \mathrm{~min}$. Unincorporated nucleotides and primers were removed by filtration with Microcon 100 microconcentrators (Amicon, Inc., Beverly, Mass.). Sequencing reactions were performed with a Prism Ready Reaction DyeDeoxy terminator cycle sequencing kit (Applied Biosystems, Inc., Foster City, Calif.). An automated DNA sequencer (model 373A; Applied Biosystems) was used to characterize both strands of the target hsp65 segment. Unambiguous sequence data were obtained for a 360 -bp region of the 441-bp fragment for all strains. The data were assembled and edited with the EDITSEQ, ALIGN, and MEGALIGN computer programs (DNASTAR Madison, Wis.). 
4455555555556666666666777777

440333567770444567999012227

3900361332524889654425870025883

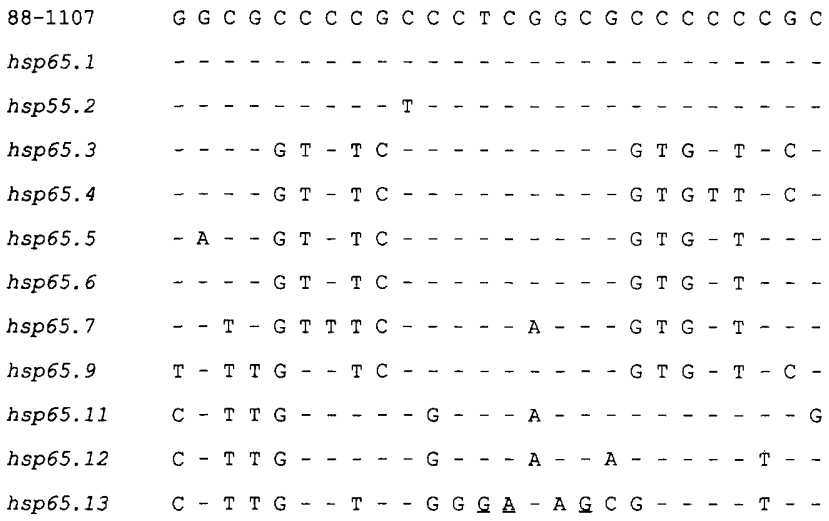

FIG. 1. Polymorphic nucleotide sites in the 360 -bp fragment of the $h s p 65$ gene sequence from 56 strains ( 11 alleles) belonging to the MAC. Nucleotide numbers are based on a previously published sequence for hsp65 of Mycobacterium tuberculosis (38) and are indicated vertically at the top. The sequences of the 11 alleles are compared with the previously published sequence of the $h s p 65$ fragment of $M$. avium 88-1107 (GenBank accession no. U17923). The underlined nucleotides are nucleotides where there were nonsynonymous changes, as described in the text. The nucleotides at sites where there was no variation are not shown.

Phylogenetic trees were constructed by performing a maximum-parsimony analysis with the computer program PAUP 3.1.1, using the branch-swapping and nearest-neighbor interchange options (42). The data were resampled with 1,000 bootstrap replications.

Calculation of the level of agreement between species identification methods. The level of agreement between species assignments as determined by the various methods was calculated by adding the number of isolates for which there was complete agreement on species assignment and dividing by the total number of isolates examined. For example, there was complete agreement in the species assignments based on seroagglutination, species-specific nucleic acid probe, $T$ catalase serotyping, and HPLC profile analyses (Table 1) for 26 of the 33 strains evaluated; these data resulted in a calculated level of agreement of $79 \%(26 / 33)$

Nucleotide sequence accession numbers. The nucleotide sequences which we determined have been deposited in the GenBank database under accession numbers U85631 through U85641.

\section{RESULTS}

Nucleotide polymorphism. Sequencing of the 360-bp $h s p 65$ fragment revealed that 11 of the 56 sequences determined were unique. All of the nucleotide variation was due to point mutations. Each distinctive sequence was given an arbitrary allele designation, such as $h s p 65.1, h s p 65.2$, etc. Phylogenetic analysis showed that these sequences grouped into clusters unique to MAC when they were compared with 94 sequences representing 25 mycobacterial species (25; data not shown). A total of 26 polymorphic nucleotide sites were identified, and three of the variants had nonsynonymous amino acid substitutions resulting in a Ser-to-Glu change at codon 133 and an Asp-to-Glu change at codon 141 in the hsp65.13 allele (Fig. 1). On average, pairs of alleles differed at $9(2.5 \%)$ of the nucleotide sites (Fig. 1). The phylogenetic analysis revealed that the 11 alleles formed three clusters, which, for ease of discussion, were designated clusters $\mathrm{A}$ through $\mathrm{C}$ (Fig. 2A)

Cluster A. A total of 30 of the 56 isolates evaluated had either allele $h s p 65.1$ or allele $h s p 65.2$ and formed cluster A on the phylogenetic tree (Fig. 2A). All 17 reference strains determined to be $M$. avium isolates based on previously reported serovar analysis or species-specific Gen-Probe hybridization results grouped in this cluster. There was restricted $h s p 65$ allelic diversity among the $M$. avium strains, and only one synonymous nucleotide substitution (a C-to-T transition at position 575) differentiated $h s p 65.1$ from $h s p 65.2$. These two alleles differed on average by 10 nucleotides (range, 7 to 15 nucleotides) from the other nine $h s p 65$ alleles (Fig. 1).

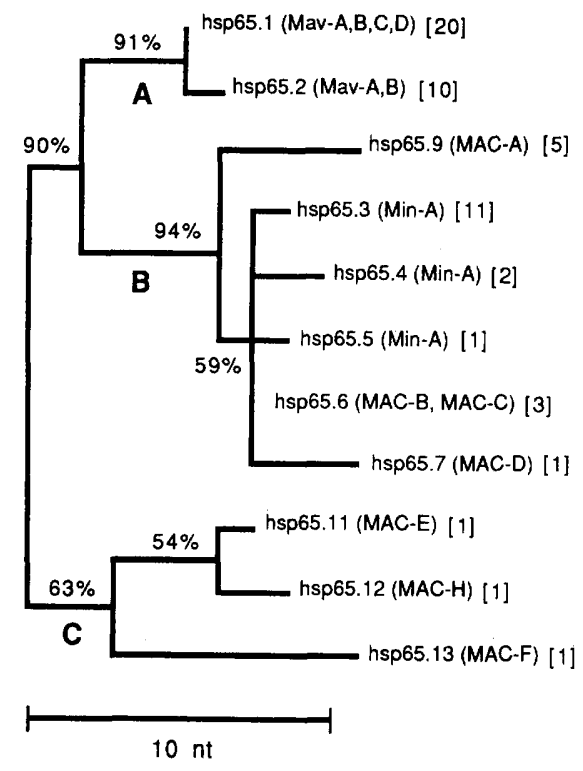

A

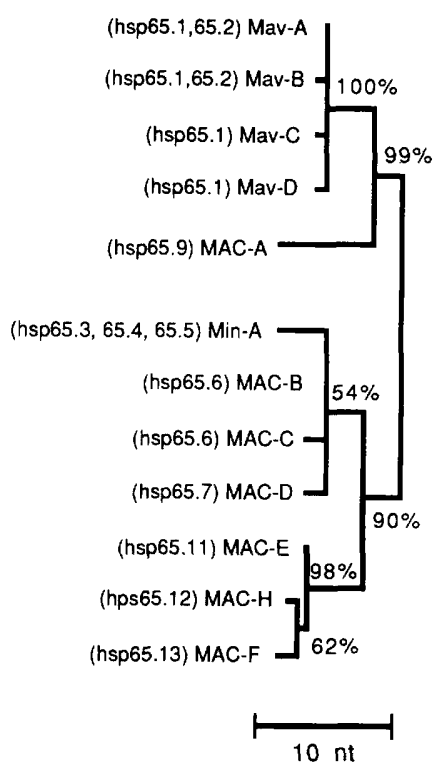

B

FIG. 2. Comparison of phylogenetic trees based on a 360-bp sequence of $h s p 65$ (A) and on a 299-bp sequence of the 16S-23S rDNA ITS (B) obtained from 56 MAC organisms. The number of strains with each allele is indicated in brackets. The horizontal lengths represent genetic distances. Scale bar $=10$ nucleotide (nt) differences Note that the trees are drawn to different scales. The percentage of bootstrap replications which yielded each group is indicated. 
Cluster B. A total of 23 strains had one of the six alleles (hsp65.3 to $h s p 65.7$ and $h s p 65.9$ ) assigned to cluster B (Fig. 2A). On average, pairs of sequences in cluster B differed at 3 $(0.8 \%)$ of the nucleotide sites and at none of the inferred amino acid positions. A total of 14 of the 15 reference strains originally identified as $M$. intracellulare had the $h s p 65.3$, $h s p 65.4$, or $h s p 65.5$ allele, and these organisms differed from one another at only one to three nucleotide sites. In the IWGMT study, no consensus species assignment was determined for reference strain Melnick (serovar 18); however, this organism had allele hsp65.7, which clustered with sequences found in isolates considered to be $M$. intracellulare (Fig. 2A). Strain 1244 Hillberry (serovar 26, hsp65.3) was allied with cluster B and was identified as an $M$. intracellulare isolate by catalase, Gen-Probe, and HPLC analyses (Table 1), although it belonged to a serovar whose species assignment was not defined.

Cluster C. A total of 3 of the 56 isolates, including 2 MAC reference strains, had one of three alleles ( $h s p 65.11$ to hsp65.13) which defined cluster $\mathrm{C}$ on the phylogenetic tree (Fig. 2A). Strain P-49 (serovar 7, hsp65.11) has a serovar considered to be expressed by $M$. intracellulare, but by consensus was identified as M. scrofulaceum in the IWGMT study (Table 1). Strain 5154-O'Connor (serovar 22, hsp65.13) belongs to a serovar whose species designation has not been determined, and the IWGMT was not able to assign it to a consensus species.

Comparison of $h s p 65$ and ITS phylogenetic trees. In the absence of significant recombination (i.e., horizontal gene transfer), phylogenetic trees constructed by using different loci of the same bacterial species should have similar overall topologies. However, if significant levels of effective recombination occur, genetic materials with very different evolutionary histories may be found in a single isolate. In such a case, the topologies of phylogenetic trees representing relationships among alleles of distinct loci may differ. Because sequence data for the 16S-23S rDNA ITS from all 56 of the MAC strains analyzed are available $(10,11)$, we were able to compare the topologies of phylogenetic trees constructed from the 360 -bp hsp65 sequence and from a 299-bp region of the $16 \mathrm{~S}-23 \mathrm{~S}$ rDNA ITS sequence (Fig. 2).

Strains with the same $h s p 65$ allele did not always have the same ITS sequence and vice versa. For example, each of the 20 $M$. avium isolates with the hsp65.1 allele had one of four ITS sequences (designated Mav-A, $-\mathrm{B},-\mathrm{C}$, and $-\mathrm{D}$ ), and the $10 \mathrm{M}$. avium isolates with the $h s p 65.2$ allele had either ITS sequence Mav-A or ITS sequence Mav-B (Fig. 2). Conversely, isolates with ITS sequence Mav-A had either the $h s p 65.1$ allele or the hsp65.2 allele. Although there was not an absolute correlation between $h s p 65$ alleles and ITS sequences, a comparison of the phylogenetic trees based on the hsp 65 alleles and the ITS region sequences revealed virtually identical topologies (Fig. 2 ). The only exception to this general pattern was strains with the hsp65.9 allele, which, based on our analysis and results reported by the IWGMT (50), were assigned to $M$. intracellulare. In contrast, the ITS sequences of these organisms (MAC-A) were similar to those of known $M$. avium strains.

Comparison of species assignments based on the results of the $h s p 65$ sequence analysis, the 16S-23S rDNA ITS sequence analysis, and the methods used by the IWGMT. To further assess the utility of species assignment and subspecific differentiation of MAC strains by a sequence-based approach, we compared the results of our analysis with the results obtained in a seroagglutination analysis, a species-specific nucleic acid probe analysis, a T-catalase serotyping analysis, and a mycolic acid HPLC analysis in the IWGMT multicenter study (50). The level of agreement among the species assignments determined by the Gen-Probe hybridization, T-catalase serotyping, and HPLC pattern analyses was $79 \%$ (Table 1). The levels of agreement for the results of these methods were $87 \%$ for strains identified by consensus as $M$. avium and $79 \%$ for strains identified by consensus as $M$. intracellulare. Strain P-49 (serovar 7, hsp65.11) was identified by consensus as M. scrofulaceum. There was insufficient agreement among the results obtained by these methods for the IWGMT to reach a consensus on species assignment for strains Melnick (serovar 18, hsp65.7) and 5154-O'Connor (serovar 22, hsp65.13).

There was $97 \%$ agreement between the phylogenetic clustering of $h s p 65$ alleles and the phylogenetic clustering of ITS sequences for all of the strains; the only difference was that isolates with the $h s p 65.9$ allele were allied more closely with $M$. intracellulare strains and the ITS sequences of these isolates (MAC-A) were allied more closely with $M$. avium strains. For the two strains not assigned to a species by consensus by the IWGMT, the results of both the hsp 65 analysis and the ITS sequence analysis unambiguously clustered strain Melnick (serovar 18 , hsp65.7) with $M$. intracellulare and strain 5154O'Connor (serovar 22, hsp65.13) with a nonspecific MAC species. The species assignments based on hsp65 and ITS sequence analysis results for the remaining 31 strains were in agreement with the IWGMT consensus species assignments in 97 and $94 \%$ of the cases examined, respectively. Strain P-49 (serovar 7, hsp65.11) was allied with non-species-specific MAC strains by $h s p 65$ and ITS sequence analyses, but was identified as $M$. scrofulaceum in the IWGMT study. However, the phylogenetic analysis grouped the ITS and hsp65 sequences of strain P-49 in a cluster unique to MAC when they were compared with the ITS and $h s p 65$ sequences of the $M$. scrofulaceum type strain and another $M$. scrofulaceum strain, Lane $3081(10$ 11, 41).

\section{DISCUSSION}

Use of $h s p 65$ sequences for species assignment and subspecific strain differentiation. Despite characterization of MAC organisms by several methods, unambiguous species assignment and subspecific differentiation of these bacteria remain problematic. Seroagglutination has been the most frequently employed method for differentiating MAC strains, but standardized antisera are not generally available. Moreover, different mycobacterial species can express the same serovar (50), and some MAC strains fail to react with available sera or spontaneously autoagglutinate and are therefore serologically nontypeable (53).

Other methods used to differentiate MAC strains include phage typing $(5,7)$, RFLP analysis of plasmids $(6,29)$, chromosomal DNA $(1,3,9,28)$, a 360-bp fragment of the $h s p 65$ gene (43), and insertion elements $(13,14,25,26,31,35,45)$, multilocus enzyme electrophoresis typing $(47,54)$, and nucleotide sequencing of the $16 \mathrm{~S}$ rDNA and ITS $(2,8,10,11,24,34$, $40,48)$. Each of these techniques has several advantages and disadvantages. For example, only $40 \%$ of MAC strains are susceptible to phage lysis, and therefore most organisms cannot be analyzed with this technique $(5,7)$. The utility of plasmid typing is restricted because only $50 \%$ of clinical isolates and $20 \%$ of environmental organisms carry plasmids (29) RFLP analysis of chromosomal DNA can discriminate well among MAC strains, but this approach cannot be used to identify strains to the species level (28). In addition, the results of RFLP analyses of chromosomal DNAs can be difficult to compare between laboratories. Although five insertion elements have been identified in MAC and used for RFLP anal- 
ysis, the lack of an insertion element common to all MAC organisms limits the utility of this technique $(13,14,25,26,31$, $35,45)$.

Recently, nucleotide sequence analyses of the 16S rDNA and the 16S-23S rDNA ITS have also been used for MAC strain differentiation $(2,8,10,11,24,34,40,48)$. The hsp 65 and ITS region sequences of 35 reference strains have been determined, but 611-bp portions of the 16S rRNA sequence have been reported for only 12 of these strains (2). We found that the variation in the 16S rDNA sequences was less than the variation in the $h s p 65$ and ITS sequences. Therefore, subspecific differentiation of MAC organisms based on 16S rDNA polymorphism was not as useful (data not shown) as differentiation based on the $h s p 65$ or ITS sequences.

Comparison of $h s p 65$ and ITS sequence variation. It is clear that MAC strains can be differentiated readily by analyzing the sequence of a portion of the $h s p 65$ gene or the $16 \mathrm{~S}-23 \mathrm{~S}$ rDNA ITS. The 56 MAC organisms whose sequences were determined by both methods were divided into 11 groups by $h s p 65$ sequencing and into 12 groups by $16 \mathrm{~S}-23 \mathrm{~S}$ rDNA ITS sequencing. On average, the nucleotide sequence variation in the ITS sequence was slightly greater than the variation in the segment of $h s p 65$ characterized for the $M$. avium strains, but the converse was true for the $M$. intracellulare strains in our sample.

Visual inspection of the branching patterns on the phylogenetic trees generated by the $h s p 65$ and ITS data did not reveal strong evidence for the occurrence of recombination. However, the results obtained when Sawyer's method (37) was used to test for nonrandom clustering of polymorphic synonymous sites in the hsp65 alleles suggested that recombination did occur. The sum of the squared condensed fragment lengths was 5,515 , and the maximum length of the condensed fragments was 21; the resulting probabilities of obtaining values equal to or greater than the observed values compared to the null distribution generated by computer sampling were 0.0050 and 0.5660 , respectively. Because these data suggest that there has been horizontal gene flow among MAC organisms, it is possible that the unusual combination of $h s p 65.9(M$. intracellulare) and ITS MAC-A (M. avium) sequences in several strains is due to recombination. Further investigation will be needed to assess the role, if any, of recombinational processes in generating genetic diversity in MAC organisms.

Comparison of species assignments by sequence-based methods and methods used by the IWGMT. A comparison of the results of our analysis and the results of the IWGMT study showed that the estimates of MAC strain relatedness were generally similar; however, some important differences were apparent. For the most part, the results obtained by the methods used by the IWGMT are in agreement with the sequencebased results for strains assigned to M. avium. However, the IWGMT data did not agree with the sequence data and were not internally consistent for the species assignments of several $M$. intracellulare and MAC strains. For example, a consensus species assignment was not reached by the IWGMT for reference strain Melnick (serovar 18); however, the sequence results for both hsp65 (hsp65.7) and ITS (MAC-D) unambiguously clustered this strain with $M$. intracellulare. The results of the partial $16 \mathrm{~S}$ rDNA sequence analysis for this isolate support a close relationship between this organism and $M$. intracellulare (2). Strain P-49 (serovar 7, hsp65.11) was designated M. scrofulaceum by the IWGMT, whereas $h s p 65$ and ITS sequence analyses aligned it with a nonspecific MAC species (Fig. 2). Likewise, the partial $16 \mathrm{~S}$ rDNA sequence obtained for this strain was unique and differed from previously published $M$. intracellulare, $M$. avium, and $M$. scrofulaceum sequences by 6,10 , and 19 nucleotides, respectively (2). The results obtained for $16 \mathrm{~S}$
rDNA sequences of other mycobacterial isolates with phenotypic properties characteristic of both the MAC and $M$. scrofulaceum did not clarify the species assignments of these organisms $(40,48)$. Several other workers have also suggested that there is a species intermediate between $M$. avium and $M$. scrofulaceum $(10,11,16,32,39,44,49)$; perhaps strain P-49 belongs to this species.

Summary. One of the primary goals of our study was to assess the level of $h s p 65$ polymorphism and thereby determine the potential utility of this characteristic for subspecific strain differentiation and species assignment. Our results demonstrate that automated sequence analysis of a 360-bp region of hsp65 is an attractive candidate for a technique that can be used for species assignment of MAC organisms. It produces rapid and unambiguous data, unlike most other differentiation approaches. The strategy is PCR based and therefore may be employed with primary samples or early positive cultures $(23$, 30a). $h s p 65$ is more variable in MAC strains than the gene encoding 16S rRNA, which has also been used as a target for sequence-based species assignment, suggesting that it has greater resolving power for molecular epidemiology studies and identification of medically important subspecific strains. In addition, sequence analysis of $h s p 65$ may provide data useful for classification of mycobacterial strains that do not belong to well-established or thoroughly characterized species.

\section{ACKNOWLEDGMENTS}

This work was supported by Public Health Service Grants AI37004 and DA09238 (to J.M.M.), AI07392 (to R.F.), and AI35230 (to R.F.) and by funds from the Durham VA Medical Center's Research Center on AIDS and HIV infection (to R.F.). J.M.M. is an Established Investigator of the American Heart Association.

\section{REFERENCES}

1. Arbeit, R. D., A. Slutsky, T. W. Barber, J. N. Maslow, S. Niemczyk, J. O. Falkinham III, G. T. O'Connor, and C. F. von Reyn. 1993. Genetic diversity among strains of Mycobacterium avium causing monoclonal and polyclonal bacteremia in patients with AIDS. J. Infect. Dis. 167:1384-1390.

2. Böddinghaus, B., J. Wolters, W. Hiekens, and E. C. Böttger. 1990. Phylogenetic analysis and identification of different serovars of Mycobacterium in tracellulare at the molecular level. FEMS Microbiol. Lett. 70:197-204.

3. Burki, D. R., C. Bernasconi, T. Bodmer, and A. Telenti. 1995. Evaluation of the relatedness of strains of Mycobacterium avium using pulsed-field gel electrophoresis. Eur. J. Clin. Microbiol. Infect. Dis. 14:212-217.

4. Chin, D. P., A. L. Reingold, E. N. Stone, E. Vittinghoff, C. R. Horsburgh, Jr., E. M. Simon, D. M. Yajko, W. K. Hadley, S. M. Ostroff, and P. C. Hopewell. 1994. The impact of Mycobacterium avium complex bacteremia and its treatment on survival of AIDS patients-a prospective study. J. Infect. Dis. 170:578-584.

5. Crawford, J. T., and J. H. Bates. 1985. Phage typing of the Mycobacterium avium-intracellulare-scrofulaceum complex: a study of strains of diverse geographic and host origin. Am. Rev. Respir. Dis. 132:386-389.

6. Crawford, J. T., and J. H. Bates. 1986. Analysis of plasmids in Mycobacterium avium-intracellulare isolates from persons with acquired immunodeficiency syndrome. Am. Rev. Respir. Dis. 134:659-661.

7. Crawford, J. T., J. K. Fitzhugh, and J. H. Bates. 1981. Phage typing of the Mycobacterium avium-intracellulare-scrofulaceum complex. Am. Rev. Respir Dis. 124:559-562.

8. De Smet, K. A. L., I. N. Brown, M. Yates, and J. Ivanyi. 1995. Ribosomal internal transcribed spacer sequences are identical among Mycobacterium avium-intracellulare complex isolates from AIDS patients, but vary among isolates from elderly pulmonary disease patients. Microbiology 141:27392747.

9. Feizabadi, M. M., I. D. Robertson, D. V. Cousins, D. Dawson, W. Chew, G. L Gilbert, and D. J. Hampson. 1996. Genetic characterization of Mycobacterium avium isolates recovered from humans and animals in Australia. Epidemiol. Infect. 116:41-49.

10. Frothingham, R., and K. H. Wilson. 1993. Sequence-based differentiation of strains in the Mycobacterium avium complex. J. Bacteriol. 175:2818-2825.

11. Frothingham, R., and K. H. Wilson. 1994. Molecular phylogeny of the Mycobacterium avium complex demonstrates clinically meaningful divisions. J. Infect. Dis. 169:305-312.

12. Fry, K. L., P. S. Meissner, and J. O. Falkinham III. 1986. Epidemiology of infection by nontuberculous mycobacteria. VI. Identification and use of 
epidemiologic markers for studies of Mycobacterium avium, M. intracellulare, and $M$. scrofulaceum. Am. Rev. Respir. Dis. 134:39-43.

13. Green, E. P., M. L. V. Tizard, M. T. Moss, J. Thompson, D. J. Winterbourne, J. J. McFadden, and J. Herman-Taylor. 1989. Sequence and characteristics of IS900, an insertion element identified in a human Crohn's disease isolate of Mycobacterium paratuberculosis. Nucleic Acids Res. 17:9063-9073.

14. Guerrero, C., C. Bernasconi, D. Burki, T. Bodmer, and A. Telenti. 1995. A novel insertion element from Mycobacterium avium, IS1245, is a specific target for analysis of strain relatedness. J. Clin. Microbiol. 33:304-307.

15. Guthertz, L. S., B. Damsker, E. J. Bottone, E. G. Ford, T. F. Midura, and J. M. Janda. 1989. Mycobacterium avium and Mycobacterium intracellulare infections in patients with and without AIDS. J. Infect. Dis. 160:1037-1041.

16. Hawkins, J. E. 1977 . Scotochromogenic mycobacteria which appear intermediate between Mycobacterium avium-intracellulare and Mycobacterium scrofulaceum. Am. Rev. Respir. Dis. 116:963-964. (Letter.)

17. Horsburgh, C. R., Jr., J. A. Havlik, D. A. Ellis, E. Kennedy, S. A. Fann, R. E Dubois, and S. E. Thompson. 1991. Survival of patients with acquired immune deficiency syndrome and disseminated Mycobacterium avium complex infection with and without antimycobacterial chemotherapy. Am. Rev. Respir. Dis. 144:557-559.

18. Horsburgh, C. R., Jr., and R. M. Selik. 1989. The epidemiology of disseminated nontuberculous mycobacterial infection in the acquired immunodeficiency syndrome (AIDS). Am. Rev. Respir. Dis. 139:4-7.

19. Hoyt, L., J. Oleske, B. Holland, and E. Connor. 1992. Nontuberculous mycobacteria in children with acquired immunodeficiency syndrome. Pediatr. Infect. Dis. J. 11:354-360.

20. Ichiyama, S., K. Shimokata, and M. Tsukamura. 1988. The isolation of Mycobacterium avium complex from soil, water, and dusts. Microbiol. Immunol. 32:733-739.

21. Inderlied, C. B., C. A. Kemper, and L. E. M. Bermudez. 1993. The Mycobacterium avium complex. Clin. Microbiol. Rev. 6:266-310.

22. Jacobson, M. A., P. C. Hopewell, D. M. Yajko, W. K. Hadley, E. Lazarus, P. K. Mohanty, G. W. Modin, D. W. Feigal, P. S. Cusick, and M. A. Sande. 1991. Natural history of disseminated Mycobacterium avium complex infection in AIDS. J. Infect. Dis. 164:994-998.

23. Kapur, V., L.-L. Li, M. R. Hamrick, B. B. Plikaytis, T. M. Shinnick, A Telenti, W. R. Jacobs, Jr., A. Banerjee, S. Cole, K. Y. Yuen, J. E. Clarridge III, B. N. Kreiswirth, and J. M. Musser. 1995. Rapid Mycobacterium species assignment and unambiguous identification of mutations associated with antimicrobial resistance in Mycobacterium tuberculosis by automated DNA sequencing. Arch. Pathol. Lab. Med. 119:131-138.

24. Kirschner, P., B. Springer, U. Vogel, A. Meier, A. Wrede, M. Kiekenbeck, F.-C. Bange, and E. C. Böttger. 1993. Genotypic identification of mycobacteria by nucleic acid sequence determination: report of a 2-year experience in a clinical laboratory. J. Clin. Microbiol. 31:2882-2889.

25. Kunze, Z. M., F. Portaels, and J. J. McFadden. 1992. Biologically distinct subtypes of Mycobacterium avium differ in possession of insertion sequence IS901. J. Clin. Microbiol. 30:2366-2372.

26. Kunze, Z. M., S. Wall, R. Appelberg, M. T. Silva, F. Portaels, and J. J. McFadden. 1991. IS901, a new member of a widespread class of atypical insertion sequences, is associated with pathogenicity in Mycobacterium avium. Mol. Microbiol. 5:2265-2272.

27. Lewis, L. L. K. M. Butler, R. N. Husson, B. U. Mueller, C. L. Fowler, S. M. Steinberg, and P. A. Pizzo. 1992. Defining the population of human immunodeficiency virus-infected children at risk for Mycobacterium avium-intracellulare infection. J. Pediatr. 121:677-683.

28. Mazurek, G. H., S. Hartman, Y. Zhang, B. A. Brown, J. S. R. Hector, D. Murphy, and R. J. Wallace, Jr. 1993. Large DNA restriction fragment polymorphism in the Mycobacterium avium-M. intracellulare complex: a potential epidemiologic tool. J. Clin. Microbiol. 31:390-394.

29. Meissner, P. S., and J. O. Falkinham III. 1986. Plasmid DNA profiles as epidemiological markers for clinical and environmental isolates of Mycobacterium avium, Mycobacterium intracellulare, and Mycobacterium scrofulaceum. J. Infect. Dis. 153:325-331.

30. Nightingale, S. D., L. T. Byrd, P. M. Southern, J. D. Jockusch, S. X. Cal, and B. A. Wynne. 1992. Incidence of Mycobacterium avium-intracellulare complex bacteremia in human immunodeficiency virus-positive patients. J. Infect. Dis. 165:1082-1085.

30a.Pai, S., N. Esen, and J. M. Musser. Routine rapid species assignment in a clinical microbiology laboratory based on species specific allelic variation in the 65-kilodalton heat shock protein gene (hsp65). Arch. Pathol. Lab. Med., in press.

31. Perez, M. H., N. G. Fomukong, T. Hellyer, I. N. Brown, and J. W. Dale. 1994. Characterization of IS 1110 , a highly mobile genetic element from Mycobacterium avium. Mol. Microbiol. 12:714-724.

32. Portaels, F. 1978. Difficulties encountered in identification of $M$. avium-M. intracellulare, M. scrofulaceum, and related strains. Am. Rev. Respir. Dis. 118:969. (Letter.)

33. Reznikov, M., J. H. Leggo, and D. J. Dawson. 1971. Investigation by seroagglutination of strains of the Mycobacterium intracellulare-M. scrofulaceum group from house dusts and sputum in southeastern Queensland. Am. Rev. Respir. Dis. 104:951-953.

34. Rogall, T., J. Wolters, T. Flohr, and E. C. Böttger. 1990. Towards a phylog eny of species at the molecular level within the genus Mycobacterium. Int. J. Syst. Bacteriol. 40:323-330.

35. Roiz, M. P., E. Palenque, C. Guerrero, and M. J. Garcia. 1995. Use of restriction fragment length polymorphism as a genetic marker for typing Mycobacterium avium strains. J. Clin. Microbiol. 33:1389-1391.

36. Saito, H., H. Tomioka, K. Sato, H. Tasaka, and D. J. Dawson. 1990. Identification of various serovar strains of Mycobacterium avium complex by using DNA probes specific for Mycobacterium avium and Mycobacterium intracellulare. J. Clin. Microbiol. 28:1694-1697.

37. Sawyer, S. 1989. Statistical tests for detecting gene conversion. Mol. Biol Evol. 6:526-538.

38. Shinnick, T. 1987. The 65-kilodalton antigen of Mycobacterium tuberculosis. J. Bacteriol. 169:1080-1088.

39. Soini, H., E. Eerola, and M. K. Viljanen. 1996. Genetic diversity among Mycobacterium avium complex Accuprobe-positive isolates. J. Clin. Microbiol. 34:55-57.

40. Springer, B., L. Stockman, K. Teschner, G. D. Roberts, and E. C. Böttger 1996. Two-laboratory collaborative study on identification of mycobacteria: molecular versus phenotypic methods. J. Clin. Microbiol. 34:296-303.

41. Swanson, D. S., X. Pan, and J. M. Musser. 1996. Identification and subspecific differentiation of Mycobacterium scrofulaceum by automated sequencin of a region of the gene ( $h s p 65$ ) encoding a 65-kilodalton heat shock protein. J. Clin. Microbiol. 34:3151-3159.

42. Swofford, D. L. 1991. PAUP: phylogenetic analysis using parsimony, version 3.1.1. Illinois Natural History Survey, Champaign.

43. Telenti, A., F. Marchesi, M. Balz, F. Bally, E. C. Böttger, and T. Bodmer 1993. Rapid identification of mycobacteria to the species level by polymerase chain reaction and restriction enzyme analysis. J. Clin. Microbiol. 31:175178.

44. Tomioka, H., H. Saito, K. Sato, H. Tasaka, and D. J. Dawson. 1993. Identification of Mycobacterium avium complex strains belonging to serovars 21-28 by three commercial DNA probe tests. Tuberc. Lung Dis. 74:91-95.

45. Via, L. E., and J. O. Falkinham III. 1993. IS 1141 and colonial variation in Mycobacterium intracellulare. GenBank accession number L10239.

46. Wallace, R. J., Jr. 1987. Nontuberculous mycobacteria and water: a love affair with increasing clinical importance. Infect. Dis. Clin. N. Am. 1:677686.

47. Wasem, C. F., C. M. McCarthy, and L. W. Murray. 1991. Multilocus enzyme electrophoresis analysis of the Mycobacterium avium complex and othe mycobacteria. J. Clin. Microbiol. 29:264-271.

48. Wayne, L. G., R. C. Good, E. C. Böttger, R. Butler, M. Dorsch, T. Ezaki, W. Gross, V. Jonas, J. Kilburn, P. Kirschner, M. I. Krichevsky, M. Ridell, T. M. Shinnick, B. Springer, E. Stackebrandt, I. Tarnok, Z. Tarnok, H. Tasaka, V. Vincent, N. G. Warren, C. A. Knott, and R. Johnson. 1996. Semantide- and chemotaxonomy-based analyses of some problematic phenotypic clusters of slowly growing mycobacteria, a cooperative study of the International Working Group on Mycobacterial Taxonomy. Int. J. Syst. Bacteriol. 46:280-297.

49. Wayne, L. G., R. C. Good, M. I. Krichevsky, Z. Blacklock, H. L. David, D. Dawson, W. Gross, J. Hawkins, V. V. Levy-Frebault, C. McManus, F. Portaels, S. Rüsch-Gerdes, K. H. Schröder, V. A. Silcox, M. Tsukamura, L. Van Den Breen, and M. A. Yakrus. 1991. Fourth report of the cooperative open-ended study of slowly growing mycobacteria by the Internationa Working Group on Mycobacterial Taxonomy. Int. J. Syst. Bacteriol. 41:463472 .

50. Wayne, L. G., R. C. Good, A. Tsang, R. Butler, D. Dawson, D. Groothuis, W Gross, J. Hawkins, J. Kilburn, M. Kubin, K. H. Schröder, V. A. Silcox, C. Smith, M.-F. Thorel, C. Woodley, and M. A. Yakrus. 1993. Serovar determination and molecular taxonomic correlation in Mycobacterium avium Mycobacterium intracellulare, and Mycobacterium scrofulaceum: a cooperative study of the International Working Group on Mycobacterial Taxonomy. Int. J. Syst. Bacteriol. 43:482-489.

51. Wayne, L. G., and H. A. Sramek. 1992. Agents of newly recognized or infrequently encountered mycobacterial diseases. Clin. Microbiol. Rev. 5:125

52. Yajko, D. M., D. P. Chin, P. C. Gonzalez, P. S. Nassos, P. C. Hopewell, A. L. Reingold, C. R. Horsburgh, Jr., M. A. Yakrus, S. M. Ostroff, and W. K. Hadley. 1995. Mycobacterium avium complex in water, food, and soil samples collected from the environment of HIV-infected individuals. J. Acquir. Immune Defic. Syndr. Hum. Retrovirol. 9:176-182.

53. Yakrus, M. A., and R. C. Good. 1990. Geographic distribution, frequency, and specimen source of Mycobacterium avium complex serotypes isolated from patients with acquired immunodeficiency syndrome. J. Clin. Microbiol. 28:926-929.

54. Yakrus, M. A., M. W. Reeves, and S. B. Hunter. 1992. Characterization of isolates of Mycobacterium avium serotypes 4 and 8 from patients with AIDS by multilocus enzyme electrophoresis. J. Clin. Microbiol. 30:1474-1478. 